\title{
A TAUBERIAN THEOREM FOR IDEAL STATISTICAL CONVERGENCE
}

\author{
MAREK BALCERZAK AND PAOLO LEONETTI
}

\begin{abstract}
Given an ideal $\mathcal{I}$ on the positive integers, a real sequence $\left(x_{n}\right)$ is said to be $\mathcal{I}$-statistically convergent to $\ell$ provided that

$$
\left\{n \in \mathbf{N}: \frac{1}{n}\left|\left\{k \leq n: x_{k} \notin U\right\}\right| \geq \varepsilon\right\} \in \mathcal{I}
$$

for all neighborhoods $U$ of $\ell$ and all $\varepsilon>0$. First, we show that $\mathcal{I}$-statistical convergence coincides with $\mathcal{J}$-convergence, for some unique ideal $\mathcal{J}=\mathcal{J}(\mathcal{I})$. In addition, $\mathcal{J}$ is Borel [analytic, coanalytic, respectively] whenever $\mathcal{I}$ is Borel [analytic, coanalytic, resp.].

Then we prove, among others, that if $\mathcal{I}$ is the summable ideal $\{A \subseteq \mathbf{N}$ : $\left.\sum_{a \in A} 1 / a<\infty\right\}$ or the density zero ideal $\left\{A \subseteq \mathbf{N}: \lim _{n \rightarrow \infty} \frac{1}{n}|A \cap[1, n]|=0\right\}$ then $\mathcal{I}$-statistical convergence coincides with statistical convergence. This can be seen as a Tauberian theorem which extends a classical theorem of Fridy. Lastly, we show that this is never the case if $\mathcal{I}$ is maximal.
\end{abstract}

\section{INTRODUCTION}

Let $\mathcal{I} \subseteq \mathcal{P}(\mathbf{N})$ be an ideal, that is, a collection of subsets of the positive integers $\mathbf{N}$ closed under taking finite unions and subsets. It is also assumed that $\mathcal{I}$ contains the collection Fin of finite subsets of $\mathbf{N}$ and that, unless otherwise stated, $\mathcal{I}$ is proper, that is, it is different from $\mathcal{P}(\mathbf{N})$. Among the most important ideals we can find the family of asymptotic density zero sets

$$
\mathcal{Z}:=\left\{A \subseteq \mathbf{N}: \lim _{n \rightarrow \infty} \frac{|A \cap[1, n]|}{n}=0\right\}
$$

and the summable ideal

$$
\mathcal{I}_{1 / n}:=\left\{S \subseteq \mathbf{N}: \sum_{n \in S} \frac{1}{n}<\infty\right\} .
$$

2010 Mathematics Subject Classification. Primary: 40A35, 11B05. Secondary: 54A20.

Key words and phrases. Ideal statistical convergence; Tauberian condition; submeasures; generalized density ideal; maximal ideals.

P.L. is supported by the Austrian Science Fund (FWF), project F5512-N26. 
Let $X$ be a Hausdorff topological space. Given an ideal $\mathcal{I}$, a sequence $\left(x_{n}\right)$ taking values in $X$ is said to be $\mathcal{I}$-convergent to $\ell \in X$, in short $x_{n} \rightarrow_{\mathcal{I}} \ell$, if

$$
\left\{n \in \mathbf{N}: x_{n} \notin U\right\} \in \mathcal{I}
$$

for all neighborhoods $U$ of $\ell$. In the literature, $\mathcal{Z}$-convergence is usually called statistical convergence, see [2, 7] and references therein. We recall that, if $\mathcal{I} \neq$ Fin and $X$ has at least two distinct points, then $\mathcal{I}$-convergence does not correspond to ordinary convergence with respect to any topology on the same base set, see [21, Example 2.2] and [19, Proposition 4.2]. In particular, the notion of ideal convergence is a "proper extension" of classical convergence.

Recently, Das and Savas introduced in [6] the notion of $\mathcal{I}$-statistical convergence: a sequence $\left(x_{n}\right)$ taking values in $X$ is said to be $\mathcal{I}$-convergent to $\ell \in X$ if

$$
\left\{n \in \mathbf{N}: \frac{\left|\left\{k \in[1, n]: x_{k} \notin U\right\}\right|}{n} \geq \varepsilon\right\} \in \mathcal{I}
$$

for all neighborhoods $U$ of $\ell$ and all $\varepsilon>0$ (note that the original definition has been given in the context of normed spaces). The aim of this article is threefold.

First, the authors of [6] remark that Fin-statistical convergence corresponds to statistical convergence, cf. also [5, Remark 1]. Hence, one may wonder whether $\mathcal{I}$-statistical convergence corresponds to $\mathcal{J}$-convergence, for some ideal $\mathcal{J}=\mathcal{J}(\mathcal{I})$. We give a positive answer, in a slightly more general context, see Theorem 2.3.

Second, the same authors claim in [6, Remark 2] that there exists a sequence $\left(x_{n}\right)$ which is $\mathcal{Z}$-statistically convergent but not statistically convergent. However, it turns out that their claim is false. Indeed, we show that $\mathcal{Z}$-statistical convergence and statistical convergence coincide, see Theorem 2.7 and Corollary 2.8. As we will explain in the next Section, this is a Tauberian theorem which extends a classical result of Fridy [13, Theorem 3]. Related results have been extensively studied in the literature, see e.g. [3, 9, 14, 15, 16, 24, 25, 26, 27, 28].

Lastly, on the opposite direction, we prove that $\mathcal{I}$-statistical convergence never coincides with statistical convergence whenever $\mathcal{I}$ is maximal, see Theorem 2.10.

\section{MAin RESUlts}

An ideal $\mathcal{I}$ is said to be a $\mathrm{P}$-ideal if it is $\sigma$-directed modulo finite sets, i.e., for each sequence $\left(A_{n}\right)$ in $\mathcal{I}$ there exists $A \in \mathcal{I}$ such that $A_{n} \backslash A$ is finite for all $n$. By identifying sets of integers with their characteristic functions, we equip $\mathcal{P}(\mathbf{N})$ with the Cantor-space topology and therefore we can assign the topological complexity to the ideals on N. In particular, we can speak about Borel ideals, analytic ideals, meager ideals, etc. It is a folklore result that the the ideals with lowest topological complexity are $F_{\sigma}$-ideals. We refer to [18] for a recent survey on ideals and filters. 
A map $\varphi: \mathcal{P}(\mathbf{N}) \rightarrow[0, \infty]$ is a submeasure provided that for all $A, B \subseteq \mathbf{N}$ : (i) $\varphi(\emptyset)=0$, (ii) $\varphi(A) \leq \varphi(B)$ if $A \subseteq B$, (iii) $\varphi(A \cup B) \leq \varphi(A)+\varphi(B)$, and (iv) $\varphi(\{n\})<\infty$ for all $n$. In addition, a submeasure $\varphi$ is lower semicontinuous if: (v) $\varphi(A)=\lim _{n \rightarrow \infty} \varphi(A \cap[1, n])$ for all $A$. By a classical result of Solecki, a (not necessarily proper) ideal $\mathcal{I}$ is an analytic P-ideal if and only if there exists a lower semicontinuous submeasure $\varphi$ such that $\mathcal{I}$ coincides with the exhaustive ideal $\operatorname{Exh}(\varphi)$ generated by $\varphi$, that is,

$$
\mathcal{I}=\operatorname{Exh}(\varphi):=\left\{A \subseteq \mathbf{N}: \lim _{n \rightarrow \infty} \varphi(A \backslash[1, n])=0\right\}
$$

and $\varphi(\mathbf{N})<\infty$, cf e.g. [10, Theorem 1.2.5].

Definition 2.1. A sequence of submeasures $\mu=\left(\mu_{n}\right)$ is said to be smooth provided that:

(s1) for all $n \in \mathbf{N}, \mu_{n}$ is supported on a nonempty set $I_{n}$;

(s2) $\lim _{n \rightarrow \infty} \mu_{n}(\{k\})=0$ for all $k \in \mathbf{N}$;

(S3) $\limsup _{n \rightarrow \infty} \mu_{n}(\mathbf{N})>0$.

In this regard, let $\mathcal{Z}_{\mu}$ be the ideal defined by

$$
\mathcal{Z}_{\mu}:=\left\{A \subseteq \mathbf{N}: \limsup _{n \rightarrow \infty} \mu_{n}\left(A \cap I_{n}\right)=0\right\} .
$$

Note that, if $\mathcal{I}$ is an ideal on $\mathbf{N}$, then there exists a smooth sequence of submeasures $\mu$ such that $\mathcal{I}=\mathcal{Z}_{\mu}$ : indeed, it is sufficient to set $\mu_{n}(A)$ equal to the characteristic function $\mathbf{1}_{\mathcal{P}(\mathbf{N}) \backslash \mathcal{I}}(A)$ for each $A \subseteq \mathbf{N}$ and $n \in \mathbf{N}$.

If, in addition, $\left(I_{n}\right)$ is a partition of $\mathbf{N}$ into finite nonempty sets then $\mathcal{Z}_{\mu}$ is a generalized density ideal, as introduced by Farah in [11, Section 2.10], cf. also [12]. Recall that every generalized density ideal is an analytic P-ideal: indeed, $\mathcal{Z}_{\mu}$ coincides with $\operatorname{Exh}\left(\varphi_{\mu}\right)$, where $\varphi_{\mu}$ is the lower semicontinuous submeasure $\sup _{k} \mu_{k}$. The class of generalized density ideals is very rich, including for example all Erdôs-Ulam ideals (among others, $\mathcal{Z}$ ), the Fubini product $\emptyset \times$ Fin, simple density ideals [1], and ideals defined in [23] by Louveau and Veličković, cf. [20, Section 2] and references therein.

Definition 2.2. Let $\mathcal{I}$ be an ideal and $\mu=\left(\mu_{n}\right)$ be a smooth sequence of submeasures. A sequence $\left(x_{n}\right)$ taking values in a Hausdorff topological space $X$ is said to be $(\mathcal{I}, \mu)$-convergent to $\ell$, shortened with $x_{n} \rightarrow_{(\mathcal{I}, \mu)} \ell$, if

$$
\left\{n \in \mathbf{N}: \mu_{n}\left(\left\{k \in \mathbf{N}: x_{k} \notin U\right\}\right) \notin V\right\} \in \mathcal{I}
$$

for each neighborhood $U$ of $\ell \in X$ and $V$ of $0 \in \mathbf{R}$.

In other words, the sequence $\left(x_{n}\right)$ is $(\mathcal{I}, \mu)$-convergent to $\ell$ if and only if

$$
\mu_{n}\left(\left\{k \in \mathbf{N}: x_{k} \notin U\right\}\right) \rightarrow_{\mathcal{I}} 0
$$


for each neighborhood $U$ of $\ell$. Moreover, it is clear that $x_{n} \rightarrow_{(\text {Fin, }, \mu)} \ell$ if and only if $x_{n} \rightarrow_{\mathcal{Z}_{\mu}} \ell$. This observation is generalized in Theorem 2.3 below.

Hereafter, let $\lambda=\left(\lambda_{n}\right)$ be the sequence of uniform probability measures on $\mathbf{N} \cap[1, n]$, that is,

$$
\lambda_{n}(A)=\frac{|A \cap[1, n]|}{n}
$$

for all $n \in \mathbf{N}$ and $A \subseteq \mathbf{N}$. Then it is easy to see that, for each ideal $\mathcal{I},(\mathcal{I}, \lambda)$ convergence corresponds with $\mathcal{I}$-statistical convergence defined in (1). Note that $(\mathcal{I}, \mu)$-convergence includes also the case of $\mathcal{I}$-lacunary statistical convergence where each $\mu_{n}$ is the uniform probability measure on $\mathbf{N} \cap\left[a_{n}, a_{n+1}\right)$ such that $\left(a_{n}\right)$ is an increasing sequence of positive integers for which $a_{n+1}-a_{n} \rightarrow \infty$, cf. [5, Definition 6].

We are ready to state our main results (all the proofs are given in Section 3). Let us start with an equivalence with the classical notion of ideal convergence.

Theorem 2.3. Let $\mathcal{I}$ be an ideal and $\mu$ be a smooth sequence of submeasures. Then there exists a unique ideal $\mathcal{J}=\mathcal{J}(\mathcal{I}, \mu)$ such that $(\mathcal{I}, \mu)$-convergence coincides with $\mathcal{J}$-convergence. In addition, $\mathcal{J}$ is proper if and only if $\mu_{n}(\mathbf{N}) \nrightarrow_{\mathcal{I}} 0$.

To be precise, we say that $(\mathcal{I}, \mu)$-convergence "coincides" with $\mathcal{J}$-convergence if, for some Hausdorff space $X$ with at least two points, every sequence $\left(x_{n}\right)$ taking values in $X$ is $(\mathcal{I}, \mu)$-convergent to $\ell \in X$ if and only if $\left(x_{n}\right)$ is $\mathcal{J}$-convergent to $\ell$.

At this point, one may ask whether there is some relationship between the pair $(\mathcal{I}, \mu)$ and the ideal $\mathcal{J}(\mathcal{I}, \mu)$ in Theorem 2.3. First of all, we prove that, under some mild conditions, $\mathcal{J}(\mathcal{I}, \mu)$ has the same topological complexity of $\mathcal{I}$.

Theorem 2.4. Let $\mathcal{I}$ be an ideal and $\mu$ be a smooth sequence of lower semicontinuous submeasures. Then the ideal $\mathcal{J}(\mathcal{I}, \mu)$ is Borel [analytic, coanalytic, respectively] whenever $\mathcal{I}$ is Borel [analytic, coanalytic, resp.].

It turns out that if the pair $(\mathcal{I}, \mu)$ is "sufficiently nice" then we can find the ideal $\mathcal{J}(\mathcal{I}, \mu)$ explicitly. To this aim, we need the following definitions.

Definition 2.5. Given a real $\alpha>0$, we say that an ideal $\mathcal{I}$ is $\alpha$-thick provided that $A \notin \mathcal{I}$ whenever there exist a real $c>0$ and infinitely many $n \in \mathbf{N}$ such that $\mathbf{N} \cap\left[n, n+c n^{\alpha}\right] \subseteq A$.

It turns out that $\mathcal{Z}$ is 1 -thick, cf. the proof of Corollary 2.8. On the other hand, if $\mathcal{I}$ is maximal ideal, then $\mathcal{I}$ is not $\alpha$-thick, for every $\alpha>0$. Indeed, exactly one among the sets $A:=\mathbf{N} \cap \bigcup_{n}\left[a_{2 n}, a_{2 n+1}\right]$ and $A^{c}$ belongs to $\mathcal{I}$, where $a_{1}:=1$ and $a_{n+1}:=2^{a_{n}}$ for all $n \in \mathbf{N}$, and such a set contains infinitely many intervals of the type $\mathbf{N} \cap\left[n, n+c n^{\alpha}\right]$, for each $c>0$. 
Definition 2.6. Given a real $\alpha>0$, we say that a sequence of submeasures $\mu=\left(\mu_{n}\right)$ is $\alpha$-flat provided that, for each $A \subseteq \mathbf{N}$, there exists a real $d=d(A)>0$ such that

$$
\left|\mu_{n+1}(A)-\mu_{n}(A)\right| \leq d / n^{\alpha}
$$

for all $n \in \mathbf{N}$.

It is easy to show that the sequence $\lambda$ defined in (2) is 1-flat, cf. the proof of Corollary 2.8 for details. More generally, for each $\alpha>0$, a family of $\alpha$-flat sequences is given as follows. Suppose that $\mu$ is smooth and each $\mu_{n}$ is a probability measure supported on $I_{n}:=\mathbf{N} \cap\left[1, \iota_{n}\right]$, where $\left(\iota_{n}\right)$ is an increasing sequence in $\mathbf{N}$ such that:

(i) $\iota_{n} \leq b n^{\beta}$ for all $n$ and some $b, \beta>0$;

(ii) $\iota_{n+1}-\iota_{n} \leq c n^{\gamma}$ for all $n$ and some $c, \gamma>0$;

(iii) $\left|\mu_{n+1}(\{k\})-\mu_{n}(\{k\})\right| \leq d / n^{\delta}$ for all $n$, all $k \leq \iota_{n}$, and some $d, \delta>0$;

(iv) $\mu_{n}(\{k\}) \leq e / n^{\eta}$ for all $n$, all $k>\iota_{n}$, and some $e, \eta>0$;

(v) $\alpha \leq \min \{\delta-\gamma, \eta-\beta\}$.

Then it is routine to check that $\mu$ is $\alpha$-flat. Similarly, if we assume for simplicity that $\iota_{n}=n$ for all $n$, then $\mathcal{Z}_{\mu}$ is $\alpha$-thick, for some $\alpha \in(0,1)$, provided that $\mu_{n}\left(\mathbf{N} \cap\left[n-c n^{\alpha}, n\right]\right) \not \rightarrow 0$ for all $c>0$. It is worth to remark that, in such cases, the ideal $\mathcal{Z}_{\mu}$ corresponds to the ideal generated by the nonnegative regular matrix $R=\left\{r_{n, k}: n, k \in \mathbf{N}\right\}$, where $r_{n, k}:=\mu_{n}(\{k\})$, cf. e.g. [4, Section 2].

With these premises, we can state the following characterization.

Theorem 2.7. Let $\nu$ and $\mu$ be two smooth sequences of submeasures such that $\mathcal{Z}_{\nu}$ is $\alpha$-thick and $\mu$ is $\alpha$-flat, for some $\alpha \in(0,1]$. Then $\mathcal{J}\left(\mathcal{Z}_{\nu}, \mu\right)=\mathcal{Z}_{\mu}$, that is, $\left(\mathcal{Z}_{\nu}, \mu\right)$-convergence coincides with $\mathcal{Z}_{\mu}$-convergence.

Roughly, Theorem 2.7 states that if the pair $(\mathcal{I}, \mu)$ is sufficiently nice, then

$$
\mu_{n}(A) \rightarrow_{\mathcal{I}} 0 \text { implies } \mu_{n}(A) \rightarrow 0
$$

for all $A \subseteq \mathbf{N}$. At the point, if $A$ is fixed, the real sequence $\left(x_{n}\right)$ defined by $x_{n}:=\mu_{n}(A)$ is arbitrary, though nonnegative. Hence, in the case $\mathcal{I}=\mathcal{Z}$ and $\left|x_{n+1}-x_{n}\right| \leq d / n$ for all $n$ and some $d>0$ (which corresponds to 1-flatness of the sequence $\mu$ relative to $A$ ), the claim (3) can be rewritten as

$$
x_{n} \rightarrow_{\mathcal{Z}} 0 \text { implies } x_{n} \rightarrow 0 .
$$

Indeed, this is a classical result of Fridy, see [13, Theorem 3]. Here, he also proves that the Tauberian condition $\left|x_{n+1}-x_{n}\right| \leq d / n$ is best possible. This has been soon extended by Maddox, in the context of strong summability for slowly oscillating sequences, see [24, 25]. A quite different Tauberian condition for Borel 
summability (related to $1 / 2$-flatness) can be found in [15]. Finally, there are related results for statistically slowly oscillating sequences [3, 26, 27, 28] and for sequences which satisfy a gap Tauberian condition $[9,16]$.

As an application of Theorem 2.7, we obtain a sufficient condition for the equivalence between statistical convergence and $\mathcal{I}$-statistical convergence.

Corollary 2.8. Let $\mathcal{I}$ be an ideal such that $\mathcal{I} \subseteq \mathcal{Z}$. Then $\mathcal{I}$-statistical convergence coincides with statistical convergence.

Since $\mathcal{Z}$ is the ideal generated the upper asymptotic density $\mathrm{d}^{\star}$ defined by

$$
\mathrm{d}^{\star}(A):=\limsup _{n \rightarrow \infty} \frac{|A \cap[1, n]|}{n}
$$

for all $A \subseteq \mathbf{N}$ (that is, $\mathcal{Z}=\left\{A \subseteq \mathbf{N}: \mathrm{d}^{\star}(A)=0\right\}$ ), it follows that Corollary 2.8 applies to all ideals $\mathcal{I}$ of the type $\left\{A \subseteq \mathbf{N}: \mu^{\star}(A)=0\right\}$, where $\mu^{\star}$ is an "upper density" on $\mathbf{N}$, in the sense of [22], such that $\mathrm{d}^{\star} \leq \mu^{\star}$ pointwise. In particular, possible choices for $\mu^{\star}$ are: the upper Banach density, the upper analytic density, the upper Pólya density, the upper Buck density, together with all upper $\alpha$-densities with $\alpha \geq 0$ (see [22] for details; cf. also [8] for the relationship between ideals and "abstract densities").

In addition, as a special instance of Corollary 2.8, we have:

Corollary 2.9. $\mathcal{I}$-statistical convergence coincides with statistical convergence if $\mathcal{I}=\mathcal{I}_{1 / n}$ or $\mathcal{I}=\emptyset \times$ Fin.

Lastly, we show that the conclusion of Corollary 2.8 cannot be strenghtened to the whole class of ideals $\mathcal{I}$. Indeed, this is never the case if $\mathcal{I}$ is maximal.

Theorem 2.10. Let $\mathcal{I}$ be a maximal ideal. Then $\mathcal{I}$-statistical convergence does not coincide with statistical convergence.

To conclude, note that the definition of $(\mathcal{I}, \mu)$-convergence depends on the choice of the sequence $\mu$. Indeed, it is possible that $(\mathcal{I}, \mu)$-convergence does not coincide with $(\mathcal{I}, \nu)$-convergence, where $\nu$ is another smooth sequence of submeasures for which $\mathcal{Z}_{\mu}=\mathcal{Z}_{\nu}$.

Example 2.11. Let $x$ be the sequence defined by $x_{n}=\mathbf{1}_{A}(n)$ for all $n \in \mathbf{N}$, where $A:=\bigcup_{n \geq 1}[(2 n) !,(2 n+1) !]$. Moreover, set $\mu_{n}=\lambda_{n}$ and $\nu_{n}=\mathrm{d}^{\star}$, as defined in (2) and (4), respectively, for all $n \in \mathbf{N}$, so that $\mathcal{Z}_{\mu}=\mathcal{Z}_{\nu}=\mathcal{Z}$. Note that $\mathrm{d}^{\star}(A)=\mathrm{d}^{\star}\left(A^{c}\right)=1$ (in particular, $x$ is not statistically convergent). Set $A_{m}:=\left\{n \in \mathbf{N}: \lambda_{n}(A) \geq 1 / m\right\}$ for each $m \in \mathbf{N}$. Then $\left(A_{m}\right)$ is an increasing sequence of sets and there exists a maximal ideal $\mathcal{I}$ containing all the $A_{m}$ 's. It 
follows that, for every $\varepsilon>0$, the set $\left\{n \in \mathbf{N}: \lambda_{n}(n) \geq \varepsilon\right\}$ is contained in some $A_{m} \in \mathcal{I}$, so that $\lambda_{n}(A) \rightarrow_{\mathcal{I}} 0$. Therefore $x_{n} \rightarrow_{(\mathcal{I}, \mu)} 1$. On the other hand,

$$
\begin{aligned}
\left\{n \in \mathbf{N}: \nu_{n}\left(\left\{k \in \mathbf{N}:\left|x_{k}-1\right|\right.\right.\right. & \geq 1 / 2\}) \geq 1 / 2\} \\
& =\left\{n \in \mathbf{N}: \nu_{n}\left(\left\{k \in \mathbf{N}: x_{k}=0\right\}\right) \geq 1 / 2\right\} \\
& =\left\{n \in \mathbf{N}: \mathrm{d}^{\star}\left(A^{c}\right) \geq 1 / 2\right\}=\mathbf{N} \notin \mathcal{I},
\end{aligned}
$$

hence $x_{n} \nrightarrow_{(\mathcal{I}, \nu)} 1$.

We leave as open questions for the interested reader to "characterize" the class of ideals $\mathcal{I}$ for which $\mathcal{I}$-statistical convergence coincides with statistical convergence and to establish whether Theorem 2.10 holds for nonmeasurable ideals or those without the Baire property.

\section{Proofs}

Before we start proving our results, we state the next lemma (which is straightforward, we omit details):

Lemma 3.1. Fix ideals $\mathcal{I}, \mathcal{J}$ on $\mathbf{N}$, let $\mu, \nu$ be two smooth sequences of submeasures, and fix $\alpha, \beta>0$. Then:

(i) $(\mathcal{I}, \mu)$-convergence implies $(\mathcal{J}, \mu)$-convergence, provided that $\mathcal{I} \subseteq \mathcal{J}$;

(ii) $(\mathcal{I}, \mu)$-convergence implies $(\mathcal{I}, \nu)$-convergence, provided that, for all $A \subseteq$ $\mathbf{N}$, it holds $\nu_{n}(A) \leq \mu_{n}(A)$ for all sufficiently large $n$;

(iii) $\mathcal{I}$ is $\alpha$-thick implies that $\mathcal{J}$ is $\alpha$-thick, provided that $\mathcal{J} \subseteq \mathcal{I}$;

(iv) $\mathcal{I}$ is $\alpha$-thick implies that $\mathcal{I}$ is $\beta$-thick, provided that $\alpha \leq \beta$;

(v) $\mu$ is $\alpha$-flat implies that $\mu$ is $\beta$-flat, provided that $\beta \leq \alpha$.

Thus, let us start with the proof of Theorem 2.3.

Proof of Theorem 2.3. Given the pair $(\mathcal{I}, \mu)$, define the family

$$
\mathcal{J}=\mathcal{J}(\mathcal{I}, \mu):=\left\{A \subseteq \mathbf{N}: \mu_{n}(A) \rightarrow_{\mathcal{I}} 0\right\} .
$$

It is clear that $\mathcal{J}$ is closed under subsets. Moreover, $\mathcal{J}$ is closed under finite unions; indeed, for all $A, B \in \mathcal{J}$ and $\varepsilon>0$, we have $\left\{n \in \mathbf{N}: \mu_{n}(A) \geq \varepsilon / 2\right\} \in \mathcal{I}$ and $\left\{n \in \mathbf{N}: \mu_{n}(B) \geq \varepsilon / 2\right\} \in \mathcal{I}$; hence

$$
\left\{n: \mu_{n}(A \cup B) \geq \varepsilon\right\} \subseteq\left\{n: \mu_{n}(A) \geq \varepsilon / 2\right\} \cup\left\{n: \mu_{n}(B) \geq \varepsilon / 2\right\} \in \mathcal{I},
$$

so that $A \cup B \in \mathcal{J}$. Since $\mu$ is a smooth sequence of submeasures, we have $\lim _{n \rightarrow \infty} \mu_{n}(\{k\}) \rightarrow 0$ for all $k \in \mathbf{N}$, by (s2). It follows that $\mu_{n}(\{k\}) \rightarrow_{\mathcal{I}} 0$, which implies that Fin $\subseteq \mathcal{J}$. This shows that $\mathcal{J}$ is an ideal on $\mathbf{N}$ and, in addition, $\mathcal{J}$ is proper if and only if $\mu_{n}(\mathbf{N}) \nrightarrow_{\mathcal{I}} 0$. At this point, let us prove that $(\mathcal{I}, \mu)$ convergence coincides with $\mathcal{J}$-convergence. Let $X$ be an Hausdorff space with 
at least two points, let us say $a$ and $b$, and let $\left(x_{n}\right)$ be a sequence in $X$ which is $(\mathcal{I}, \mu)$-convergent to some $\ell \in X$, that is, $\mu_{n}\left(\left\{k \in \mathbf{N}: x_{k} \notin U\right\}\right) \rightarrow_{\mathcal{I}} 0$ for each neighborhood $U$ of $\ell$. By the definition of $\mathcal{J}$ in (5), this is equivalent to $\left\{k \in \mathbf{N}: x_{k} \notin U\right\} \in \mathcal{J}$ for each neighborhood $U$ of $\ell$, i.e., $x_{n} \rightarrow_{\mathcal{J}} \ell$. Finally, let us suppose for the sake of contradiction that there exists another ideal $\mathcal{J}^{\prime} \neq \mathcal{J}$ such that $(\mathcal{I}, \mu)$-convergence coincides with $\mathcal{J}^{\prime}$-convergence. In particular, there exists $A \in \mathcal{J} \triangle \mathcal{J}^{\prime}$ and $\mathcal{J}$-convergence coincides with $\mathcal{J}^{\prime}$-convergence. Let $\left(x_{n}\right)$ be the sequence defined by $x_{n}=a$ if $n \in A$ and $x_{n}=b$ otherwise. It follows that exactly one of the conditions $x_{n} \rightarrow_{\mathcal{J}} b$ and $x_{n} \rightarrow_{\mathcal{J}^{\prime}} b$ is true. This shows that $\mathcal{J}$ is unique, completing the proof.

Note that the ideal $\mathcal{J}(\mathcal{I}, \mu)$ defined in (5) corresponds to $\mathcal{Z}_{\mu}$ if $\mathcal{I}=$ Fin.

Proof of Theorem 2.4. Let us rewrite the ideal $\mathcal{J}(\mathcal{I}, \mu)$ defined in (5) as follows:

$$
\mathcal{J}(\mathcal{I}, \mu)=\left\{A \subseteq \mathbf{N}: \forall m \in \mathbf{N}, f_{m}(A) \in \mathcal{I}\right\},
$$

where, for each $m \in \mathbf{N}, f_{m}: \mathcal{P}(\mathbf{N}) \rightarrow \mathcal{P}(\mathbf{N})$ is the function defined by

$$
f_{m}(A):=\left\{n \in \mathbf{N}: \mu_{n}(A)>1 / m\right\}
$$

for all $A \subseteq \mathbf{N}$. At this point, by the lower semicontinuity of each $\mu_{n}$, we obtain $f_{m}(A)=\bigcup_{k \in \mathbf{N}} f_{m, k}(A)$, where, for each $k \in \mathbf{N}, f_{m, k}: \mathcal{P}(\mathbf{N}) \rightarrow \mathcal{P}(\mathbf{N})$ is the function defined by

$$
f_{m, k}(A):=\left\{n \in \mathbf{N}: \mu_{n}(A \cap[1, k])>1 / m\right\}
$$

for all $A \subseteq \mathbf{N}$ and $m \in \mathbf{N}$. Therefore

$$
\mathcal{J}(\mathcal{I}, \mu)=\left\{A \subseteq \mathbf{N}: \forall m \in \mathbf{N}, \exists k \in \mathbf{N}, f_{m, k}(A) \in \mathcal{I}\right\}=\bigcap_{m \in \mathbf{N}} \bigcup_{k \in \mathbf{N}} f_{m, k}^{-1}[\mathcal{I}] .
$$

The claim follows by noting that $f_{m, k}$ is continuous and that the continuous preimage of Borel [analytic, coanalytic, respectively] sets is Borel [analytic, coanalytic, resp.], cf. e.g. [29].

To prove the next result, we need the following intermediate lemma.

Lemma 3.2. Let $\mu$ and $\nu$ be two smooth sequences of submeasures. Then $\mathcal{Z}_{\mu^{-}}$ convergence implies $\left(\mathcal{Z}_{\nu}, \mu\right)$-convergence.

Proof. Let $\left(x_{n}\right)$ be a sequence in a Hausdorff space $X$, fix $\ell \in X$, and suppose that $x_{n} \not{ }_{\left(\mathcal{Z}_{\nu}, \mu\right)} \ell$. Then there exist a neighborhood $U$ of $\ell$ and a real $\varepsilon>0$ such that

$$
\left\{n \in \mathbf{N}: \mu_{n}\left(\left\{k \in \mathbf{N}: x_{k} \notin U\right\}\right) \geq \varepsilon\right\} \notin \mathcal{Z}_{\nu}
$$

that is,

$$
\limsup _{t \rightarrow \infty} \nu_{t}\left(\left\{n \in \mathbf{N}: \mu_{n}\left(\left\{k \in \mathbf{N}: x_{k} \notin U\right\}\right) \geq \varepsilon\right\}\right)>0
$$


It follows that there exists a strictly increasing sequence of positive integers $\left(t_{m}\right)$ and a real $\delta>0$ such that

$$
\nu_{t_{m}}\left(\left\{n \in I_{t_{m}}: \mu_{n}\left(\left\{k \in I_{n}: x_{k} \notin U\right\}\right) \geq \varepsilon\right\}\right) \geq \delta
$$

for all $m \in \mathbf{N}$. At this point, fix an integer $n_{1} \in I_{t_{1}}$ such that $\mu_{n_{1}}\left(\left\{k \in I_{n_{1}}: x_{k} \notin\right.\right.$ $U\}) \geq \varepsilon$ and define recursively a sequence $\left(n_{h}\right)$ of positive integers as follows: for each $h \in \mathbf{N}$, let $n_{h+1}$ be an integer greater than $n_{h}$ such that $\mu_{n_{h+1}}\left(\left\{k \in I_{n_{h+1}}\right.\right.$ : $\left.\left.x_{k} \notin U\right\}\right) \geq \varepsilon$; note that such an integer exists because the sequence $\left(t_{m}\right)$ is infinite and $F:=\left\{n_{1}, \ldots, n_{h}\right\} \in$ Fin $\subseteq \mathcal{Z}_{\nu}$ so that the sequence $\nu_{n}(F)$ converges to 0 and, in particular, it is smaller than $\delta$ whenever $n$ is sufficiently large. It follows that

$$
\mu_{n_{h}}\left(\left\{k \in I_{n_{h}}: x_{k} \notin U\right\}\right) \geq \varepsilon
$$

for all $h \in \mathbf{N}$, which implies $\left\{k \in \mathbf{N}: x_{k} \notin U\right\} \notin \mathcal{Z}_{\mu}$. Therefore $\left(x_{n}\right) \not_{\mathcal{Z}_{\mu}} \ell$.

We proceed now to the proof of Theorem 2.7.

Proof of Theorem 2.7. On the one hand, thanks to Lemma 3.2, $\mathcal{Z}_{\mu}$-convergence implies $\left(\mathcal{Z}_{\nu}, \mu\right)$-convergence. Conversely, let $\left(x_{n}\right)$ be a sequence in a Hausdorff space $X$ which is not $\mathcal{Z}_{\mu}$-convergent to $\ell \in X$; hence there exist a neighborhood $U$ of $\ell$, a real $\varepsilon>0$, and an increasing sequence of positive integers $\left(n_{t}\right)$ such that

$$
\mu_{n_{t}}\left(\left\{k \in \mathbf{N}: x_{k} \notin U\right\}\right) \geq \varepsilon
$$

for all $t \in \mathbf{N}$. At this point, fix $S \subseteq \mathbf{N}$. Since the sequence $\mu$ is $\alpha$-flat, we obtain that there exists $d=d(S)>0$ such that $f_{S}(n) \leq d / n^{\alpha}$ for all $n \in \mathbf{N}$, where $f_{S}(n):=\left|\mu_{n}(S)-\mu_{n+1}(S)\right|$ for each $n \in \mathbf{N}$.

Claim 1. Fix $S \subseteq \mathbf{N}$. There exists a constant $\kappa=\kappa(\alpha, d(S))>0$ such that

$$
\sum_{i=1}^{\left\lfloor c n^{\alpha}\right\rfloor} f_{S}(n+i) \leq \kappa c
$$

for all $n \in \mathbf{N}$ and reals $c>0$.

Proof. First of all, for every $n \in \mathbf{N}$ and $c>0$, we have the following upper bound

$$
\sum_{i=1}^{\left\lfloor c n^{\alpha}\right\rfloor} f_{S}(n+i) \leq \sum_{i=1}^{\left\lfloor c n^{\alpha}\right\rfloor} \frac{d}{(n+i)^{\alpha}} \leq \int_{0}^{c n^{\alpha}} \frac{d}{(n+t)^{\alpha}} \mathrm{d} t .
$$


Hence, if $\alpha \in(0,1)$, we have

$$
\begin{aligned}
\sum_{i=1}^{\left\lfloor c n^{\alpha}\right\rfloor} f_{S}(n+i) & \leq \frac{d}{1-\alpha}\left(\left(n+c n^{\alpha}\right)^{1-\alpha}-n^{1-\alpha}\right) \\
& \leq \frac{d}{1-\alpha} n^{1-\alpha}\left(\left(1+c n^{\alpha-1}\right)^{1-\alpha}-1\right) \\
& \leq \frac{d}{1-\alpha} n^{1-\alpha} \cdot c n^{\alpha-1}=\frac{c d}{1-\alpha} .
\end{aligned}
$$

Similarly, if $\alpha=1$, it follows by (7) that

$$
\sum_{i=1}^{\left\lfloor c n^{\alpha}\right\rfloor} f_{S}(n+i) \leq d \log \left(\frac{n+c n}{n}\right)=d(\log (1+c)) \leq c d,
$$

which completes the proof.

Claim 2. Fix $S \subseteq \mathbf{N}$. Then for all $\delta>0$, there exist $c>0$ and $n_{0} \in \mathbf{N}$ such that

$$
\left|\mu_{n+m}(S)-\mu_{n}(S)\right| \leq \delta
$$

for all integers $n \geq n_{0}$ and all integers $m \in\left[0, c n^{\alpha}\right]$.

Proof. With the same notation above, it follows by Claim 1 that for all $n \in \mathbf{N}$, all $c>0$, and all integers $m \in\left[1, c n^{\alpha}\right]$, we have that

$$
\left|\mu_{n+m}(S)-\mu_{n}(S)\right| \leq \sum_{i=0}^{m} f_{S}(n+i) \leq f_{S}(n)+\kappa c \leq \frac{d}{n^{\alpha}}+\kappa c .
$$

At this point, the wanted inequality (8) is obtained by choosing $c>0$ sufficiently small and $n_{0} \in \mathbf{N}$ sufficiently large so that $d / n_{0}^{\alpha}+\kappa c \leq \delta$.

To conclude, choosing $\delta=\varepsilon / 2$ and $S=\left\{k \in \mathbf{N}: x_{k} \notin U\right\}$ in Claim 2 and using inequality (6), we obtain that there exist $c>0$ and $t_{0} \in \mathbf{N}$ such that

$$
\mu_{n_{t}+m}(S) \geq \mu_{n_{t}}(S)-\varepsilon / 2 \geq \varepsilon / 2
$$

for all integers $t \geq t_{0}$ and all integers $m \in\left[0, c n_{t}^{\alpha}\right]$. Therefore

$$
A:=\left\{n \in \mathbf{N}: \mu_{n}(S) \geq \varepsilon / 2\right\} \supseteq \bigcup_{t \geq t_{0}}\left(\mathbf{N} \cap\left[n_{t},(1+c) n_{t}^{\alpha}\right]\right) .
$$

Since $\mathcal{Z}_{\nu}$ is $\alpha$-thick, then $A \notin \mathcal{Z}_{\nu}$. This implies that $x_{n} \nrightarrow_{\left(\mathcal{Z}_{\nu}, \mu\right)} \ell$.

Proof of Corollary 2.8. Let $\lambda$ be the smooth sequence of submeasures defined in (2). Thanks to Theorem 2.7, it is sufficient to show that $\mathcal{Z}=\mathcal{Z}_{\lambda}$ is 1-flat and that $\mathcal{I}$ is 1-thick. To this aim, note that, for all $A \subseteq \mathbf{N}$ and $n \in \mathbf{N}$, we have

$$
\left|\lambda_{n}(A)-\lambda_{n+1}(A)\right|=\left|\lambda_{n}(A)-\frac{\mathbf{1}_{A}(n+1)+n \lambda_{n}(A)}{n+1}\right| \leq \frac{1}{n+1} \leq \frac{1}{n} .
$$


Hence $\mathcal{Z}$ is 1 -flat. Moreover, we claim that $\mathcal{Z}$ is 1 -thick. Indeed, fix $A \subseteq \mathbf{N}$ such that there exist $c>0$ and infinitely many $n \in \mathbf{N}$ for which $\mathbf{N} \cap[n,(1+c) n] \subseteq A$. Then the upper asymptotic density of $A$ is at least $c / 1+c>0$. Hence $A \notin \mathcal{Z}$. Therefore $\mathcal{I}$ is 1 -thick by Lemma 3.1(iii).

Proof of Corollary 2.9. It is known $\mathcal{I}_{1 / n} \subseteq \mathcal{Z}$, hence the claim follows by Corollary 2.8. Similarly, it is sufficient to prove that there exists a copy of the Fubini product $\mathcal{I}=\emptyset \times$ Fin on $\mathbf{N}$ which is contained in $\mathcal{Z}$. Thus, note that $\mathcal{I}$ can be written as

$$
\left\{A \subseteq \mathbf{N}: \forall k \in \mathbf{N},\left\{n \in A: v_{2}(n)=k-1\right\} \in \mathrm{Fin}\right\},
$$

where $v_{2}(n)$ is the biggest exponent $m \in \mathbf{N}$ such that $2^{m}$ divides $n$. Fix $A \in \mathcal{I}$. Then, for each $k \in \mathbf{N}$, there exists a finite set $F=F(k) \subseteq \mathbf{N}$ such that $A \backslash F$ contains only multiples of $2^{k}$, so that the upper asymptotic density of $A$ is most $1 / 2^{k}$. By the arbitrariness of $k$, we conclude that $A \in \mathcal{Z}$. Therefore $\mathcal{I} \subseteq \mathcal{Z}$.

Proof of Theorem 2.10. Let us suppose that $\mathcal{I}$ is a maximal ideal on $\mathbf{N}$. Thanks to Theorem 2.3, there exists a unique ideal $\mathcal{J}$ such that $(\mathcal{I}, \lambda)$-convergence coincides with $\mathcal{J}$-convergence; in addition, $\mathcal{J}:=\left\{A \subseteq \mathbf{N}: \lambda_{n}(A) \rightarrow_{\mathcal{I}} 0\right\}$, see (5). To conclude, we show that $\mathcal{J} \neq \mathcal{Z}$ by proving that they have different topological complexities. To this aim, it is sufficient to prove that $\mathcal{J}$ is nonmeasurable (on the other hand, it is known that $\mathcal{Z}$ is a $F_{\sigma \delta}$-ideal).

Identifying each set $A \subseteq \mathbf{N}$ with the sequence $\left(\mathbf{1}_{A}(n): n \in \mathbf{N}\right) \in\{0,1\}^{\mathbf{N}}$, we can rewrite $\mathcal{J}$ as

$$
\mathcal{J}=\left\{x \in\{0,1\}^{\mathbf{N}}: \frac{1}{n} \sum_{i \leq n} x_{i} \rightarrow_{\mathcal{I}} 0\right\} .
$$

Claim 3. There exists an increasing sequence $\left(a_{n}\right)$ in $\mathbf{N}$ such that $a_{n} / a_{n+1} \rightarrow 0$ as $n \rightarrow \infty$ and $\bigcup_{n \in \mathbf{N}} A_{3 n-1} \notin \mathcal{I}$, where $A_{n}:=\mathbf{N} \cap\left(a_{n-1}, a_{n}\right]$ and $a_{0}:=0$.

Proof. Fix an increasing sequence $\left(a_{n}\right)$ in $\mathbf{N}$ such that $a_{n} / a_{n+1} \rightarrow 0$ and, for each $i \in\{0,1,2\}$, define $R_{i}:=\bigcup_{n \equiv i \bmod 3} A_{n}$. Since $\mathcal{I}$ is maximal, there exists a unique $i_{\star} \in\{0,1,2\}$ such that $R_{i_{\star}} \notin \mathcal{I}$. If $i_{\star}=2$ we are done. If $i_{\star}=0\left[i_{\star}=1\right.$, respectively], just delete one element [two elements, resp.] from the sequence.

At this point, fix an increasing sequence $\left(a_{n}\right)$ as in Claim 3 and define the function $\Lambda:\{0,1\}^{\mathbf{N}} \rightarrow\{0,1\}^{\mathbf{N}}$ by

$$
\Lambda(x)=\left(x_{a_{3 n-1}}: n \in \mathbf{N}\right)
$$

for each sequence $\{0,1\}^{\mathbf{N}}$. Moreover, define the function $f: \mathbf{N} \rightarrow \mathbf{N}$ such that $f(n)=k$ whenever $n \in A_{k}$, and set

$$
\begin{aligned}
\mathcal{F}:=\left\{x \in\{0,1\}^{\mathbf{N}}: x_{i}=x_{j}\right. & \text { whenever } f(i)=f(j), \\
& \text { and } \left.x_{a_{3 n-2}}=x_{a_{3 n-1}} \text { and } x_{a_{3 n}}=0 \text { for all } n \in \mathbf{N}\right\},
\end{aligned}
$$


that is, $\mathcal{F}$ is the sequence of $\{0,1\}$-valued sequences which are constant on all intervals $A_{3 n}$ and $A_{3 n-1} \cup A_{3 n-2}$, with value 0 on all the $A_{3 n}$ 's. Then the restriction of $\Lambda$ on $\mathcal{F}$ is an homeomorphism $\mathcal{F} \rightarrow\{0,1\}^{\mathbf{N}}$. Since $\mathcal{F}$ is closed, it is sufficient to show that $\Lambda(\mathcal{J} \cap \mathcal{F})$ is not measurable.

Claim 4. $\lim _{n \rightarrow \infty}\left(x_{a_{n}}-\frac{1}{a_{n}} \sum_{i \leq a_{n}} x_{i}\right)=0$ for all $x \in \mathcal{F}$.

Proof. Considering that $x_{i}=x_{j}$ whenever $f(i)=f(j)$ and that $a_{n} / a_{n+1} \rightarrow 0$ as $n \rightarrow \infty$, we have that

$$
\begin{aligned}
\frac{1}{a_{n}} \sum_{i \leq a_{n}} x_{i} & =\frac{1}{a_{n}} \sum_{i \leq n} x_{a_{i}}\left|A_{i}\right| \\
& =\left(1-\frac{a_{n-1}}{a_{n}}\right) x_{a_{n}}+\frac{1}{a_{n}} \sum_{i \leq n-1} x_{a_{i}}\left|A_{i}\right| .
\end{aligned}
$$

Therefore

$$
\begin{aligned}
\left|x_{a_{n}}-\frac{1}{a_{n}} \sum_{i \leq a_{n}} x_{i}\right| & =\left|\frac{a_{n-1}}{a_{n}} x_{a_{n}}-\frac{1}{a_{n}} \sum_{i \leq n-1} x_{a_{i}}\right| A_{i}|| \\
& \leq \frac{a_{n-1}}{a_{n}}+\frac{1}{a_{n}} \sum_{i \leq n-1}\left|A_{i}\right|=2 \frac{a_{n-1}}{a_{n}} \rightarrow 0,
\end{aligned}
$$

which completes the proof.

Lastly, we define the function $g: \mathbf{N} \rightarrow \mathbf{N}$ by $g(n):=\left\lceil\frac{n}{3}\right\rceil$ for all $n \in \mathbf{N}$ (so that $g(f(n))=k$ if $\left.n \in A_{3 k-2} \cup A_{3 k-1} \cup A_{3 k}\right)$, and set

$$
\mathcal{S}:=\left\{x \in\{0,1\}^{\mathbf{N}}: x_{n} \rightarrow_{\mathcal{U}} 0\right\}
$$

where $\mathcal{U}:=\left\{f^{-1}\left[g^{-1}[A]\right]: A \in \mathcal{I}\right\}$ (note that $\mathcal{U}$ is a maximal ideal on $\mathbf{N}$ ).

Claim 5. $\Lambda(\mathcal{J} \cap \mathcal{F})=\mathcal{S}$.

Proof. First, we show that $\mathcal{S} \subseteq \Lambda(\mathcal{J} \cap \mathcal{F})$. Fix a sequence $y \in \mathcal{S}$ and set $U:=$ $\left\{n \in \mathbf{N}: y_{n}=1\right\}$. We need to prove that there exists $x \in \mathcal{J} \cap \mathcal{F}$ such that $\Lambda(x)=y$. Note that there is exactly one sequence $x \in \mathcal{F}$ such that $\Lambda(x)=y$, that is, the unique one in $\mathcal{F}$ such that $x_{a_{3 n-1}}=y_{n}$ for all $n \in \mathbf{N}$. Let us show that $x \in \mathcal{J}$, i.e., $\frac{1}{n} \sum_{i \leq n} x_{i} \rightarrow_{\mathcal{I}} 0$. Since $y \in S$, then $U \in \mathcal{U}$, hence

$$
\tilde{U}:=f^{-1}\left[g^{-1}[U]\right]=\bigcup_{u \in U}\left(A_{3 u} \cup A_{3 u-1} \cup A_{3 u-2}\right) \in \mathcal{I} .
$$

This implies, thanks to Claim 4 (see also Figure 1 below), that, for each $\varepsilon>0$, there exists a finite set $F_{\varepsilon} \in$ Fin such that

$$
\left\{n \in \mathbf{N}: \frac{1}{n} \sum_{i \leq n} x_{i} \geq \varepsilon\right\} \subseteq F_{\varepsilon} \cup \tilde{U} \in \mathcal{I} .
$$

Therefore $x \in \mathcal{J} \cap \mathcal{F}$ and $\Lambda(x)=y$. 
Conversely, let us prove that $\Lambda(\mathcal{J} \cap \mathcal{F}) \subseteq \mathcal{S}$. Fix a sequence $x \in \mathcal{J} \cap \mathcal{F}$. Then we need to show that $\Lambda(x) \in \mathcal{S}$, that is, $x_{a_{3 n-1}} \rightarrow_{\mathcal{U}} 0$. This is equivalent to $V:=\left\{n \in \mathbf{N}: x_{a_{3 n-1}}=1\right\} \in \mathcal{U}$ and also, by the definition of $\mathcal{U}$, to

$$
\tilde{V}:=f^{-1}\left[g^{-1}[V]\right]=\bigcup_{v \in V}\left(A_{3 v} \cup A_{3 v-1} \cup A_{3 v-2}\right) \in \mathcal{I} .
$$

By assumption we know $\frac{1}{n} \sum_{i \leq n} x_{i} \rightarrow_{\mathcal{I}} 0$ and the sequence $x$ is constant on each interval $A_{n}$, with $x_{a_{3 n}}=0$ and $x_{a_{3 n-1}}=x_{a_{3 n-2}}$ for all $n$. Fix a sufficiently small $\varepsilon>0$, hence $M:=\left\{n \in \mathbf{N}: \frac{1}{n} \sum_{i \leq n} x_{i} \geq \varepsilon\right\} \in \mathcal{I}$. Thanks to Claim 4, we obtain

$$
G:=\left(\bigcup_{v \in V} A_{3 v-1}\right) \backslash M \in \text { Fin. }
$$

Therefore, thanks to Claim 3, we conclude that

$$
\tilde{V} \subseteq\left(\bigcup_{n \neq 2 \bmod 3} A_{n}\right) \cup \bigcup_{v \in V} A_{3 v-1} \subseteq\left(\bigcup_{n \neq 2 \bmod 3} A_{n}\right) \cup M \cup G \in \mathcal{I},
$$

which completes the proof.

Identifying $\{0,1\}$ with the additive group $\mathbf{Z}_{2}$, we have that $\mathcal{S}$ is a subgroup of the compact group $\{0,1\}^{\mathbf{N}}$. Moreover, $\mathcal{S}$ is not closed $\left(\right.$ since $\mathbf{1}_{\{1, \ldots, k\}}(n) \rightarrow_{\mathcal{U}} 0$ for all $k \in \mathbf{N}$ but $\mathbf{1}_{\mathbf{N}}(n) t_{\mathcal{U}} 0$ ) and it has finite index (since $\mathcal{U}$ is a maximal ideal, then there are exactly two cosets of $\mathcal{S}$ ). It follows by [17, Proposition 1.1(c)] that $\mathcal{S}$ is nonmeasurable. Thus, thanks to Claim $5, \Lambda(\mathcal{J} \cap \mathcal{F})$ is nonmeasurable.

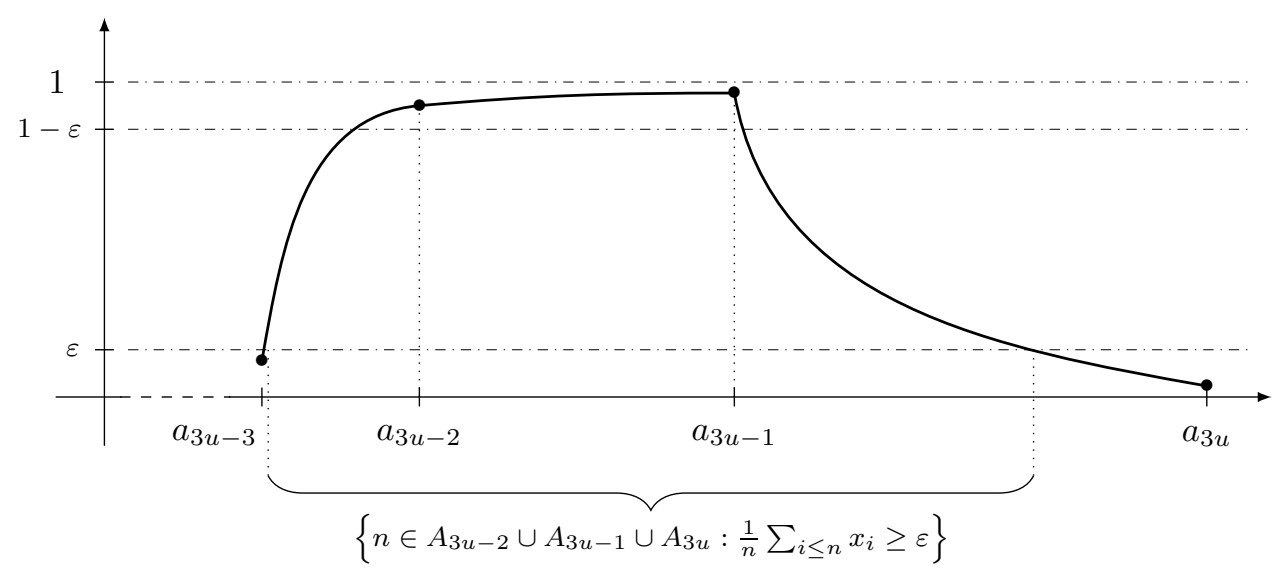

FIGURE 1. Graph of the sequence $\left(\frac{1}{n} \sum_{i \leq n} x_{i}\right)$ in the case $x_{a_{3 u-1}}=1$.

\section{REFERENCES}

1. M. Balcerzak, P. Das, M. Filipczak, and J. Swaczyna, Generalized kinds of density and the associated ideals, Acta Math. Hungar. 147 (2015), no. 1, 97-115. 
2. B. T. Bilalov and T. Y. Nazarova, Statistical convergence of functional sequences, Rocky Mountain J. Math. 45 (2015), no. 5, 1413-1423.

3. C.-P. Chen and C.-T. Chang, Tauberian conditions under which the original convergence of double sequences follows from the statistical convergence of their weighted means, J. Math. Anal. Appl. 332 (2007), no. 2, 1242-1248.

4. P. Das, R. Filipów, and J. Tryba, A note of nonregular matrices and ideals associated with them, Colloq. Math., to appear.

5. P. Das, S. Kr. Ghosal, and E. Savas, On generalizations of certain summability methods using ideals, Appl. Math. Lett. 24 (2011), no. 9, 1509-1514.

6. P. Das and E. Savas, A generalized statistical convergence via ideals, Appl. Math. Lett. 24 (2011), no. 6, 826-830.

7. G. Di Maio and L. D. R. Kočinac, Statistical convergence in topology, Topology Appl. 156 (2008), no. 1, 28-45.

8. M. Di Nasso and R. Jin, Abstract densities and ideals of sets, Acta Arith. 185 (2018), no. 4, 301-313.

9. H. Dutta and S. Das, On variations via statistical convergence, J. Math. Anal. Appl. 472 (2019), no. 1, 133-147.

10. I. Farah, Analytic quotients: theory of liftings for quotients over analytic ideals on the integers, Mem. Amer. Math. Soc. 148 (2000), no. 702, xvi+177.

11. 999-1033.

12. —_ Analytic Hausdorff gaps. II. The density zero ideal, Israel J. Math. 154 (2006), $235-246$.

13. J. A. Fridy, On statistical convergence, Analysis 5 (1985), no. 4, 301-313.

14. J. A. Fridy and M. K. Khan, Tauberian theorems via statistical convergence, J. Math. Anal. Appl. 228 (1998), no. 1, 73-95.

15. Statistical extensions of some classical Tauberian theorems, Proc. Amer. Math. Soc. 128 (2000), no. 8, 2347-2355.

16. __ Statistical gap Tauberian theorems in metric spaces, J. Math. Anal. Appl. 282 (2003), no. 2, 744-755.

17. S. Hernández, K. H. Hofmann, and S. A. Morris, Nonmeasurable subgroups of compact groups, J. Group Theory 19 (2016), no. 1, 179-189.

18. M. Hrušák, Combinatorics of filters and ideals, Set theory and its applications, Contemp. Math., vol. 533, Amer. Math. Soc., Providence, RI, 2011, pp. 29-69.

19. P. Kostyrko, T. Šalát, and W. Wilczyński, I-convergence, Real Anal. Exchange 26 (2000/01), no. 2, 669-685.

20. P. Leonetti, Limit points of subsequences, Topology Appl. 263 (2019), 221-229.

21. P. Leonetti and F. Maccheroni, Characterizations of ideal cluster points, Analysis (Berlin) 39 (2019), no. 1, 19-26.

22. P. Leonetti and S. Tringali, On the notions of upper and lower density, Proc. Edinb. Math. Soc., to appear.

23. A. Louveau and B. Veličković, A note on Borel equivalence relations, Proc. Amer. Math. Soc. 120 (1994), no. 1, 255-259.

24. I. J. Maddox, Statistical convergence in a locally convex space, Math. Proc. Cambridge Philos. Soc. 104 (1988), no. 1, 141-145. 
25. _ A Tauberian theorem for statistical convergence, Math. Proc. Cambridge Philos. Soc. 106 (1989), no. 2, 277-280.

26. F. Móricz, Tauberian conditions, under which statistical convergence follows from statistical summability $(C, 1)$, J. Math. Anal. Appl. 275 (2002), no. 1, 277-287.

27. _ Tauberian theorems for double sequences that are statistically summable $(C, 1,1)$, J. Math. Anal. Appl. 286 (2003), no. 1, 340-350.

28. _ Ordinary convergence follows from statistical summability $(C, 1)$ in the case of slowly decreasing or oscillating sequences, Colloq. Math. 99 (2004), no. 2, 207-219.

29. S. M. Srivastava, A course on Borel sets, Graduate Texts in Mathematics, vol. 180, SpringerVerlag, New York, 1998.

Institute of Mathematics, Lodz University of Technology, Ul. Wólczańska 215, 93-005 Lodz, PolAnd

E-mail address: marek.balcerzak@p.lodz.pl

Institute of Analysis and Number Theory, Graz University of Technology, Kopernikusgasse 24/II, 8010 Graz, Austria

E-mail address: leonetti.paolo@gmail.com 